\title{
Cardiac Rehabilitation Inventory (CRI)
}

Please indicate the extent to which you agree with each of the following statements by clearly marking one of the circles. There are no right or wrong answers so please be as honest as possible and give a response for all statements. Thank you.

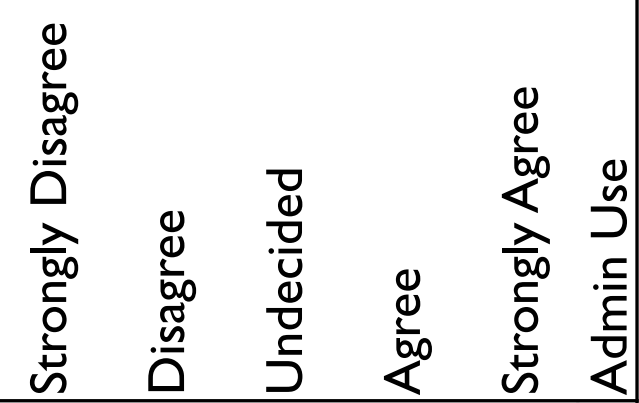

I do not think I will benefit that much from cardiac rehabilitation

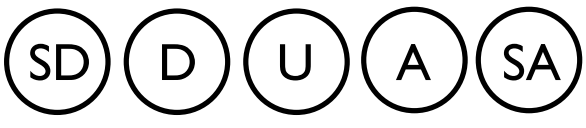

2 I would not feel safe exercising alone

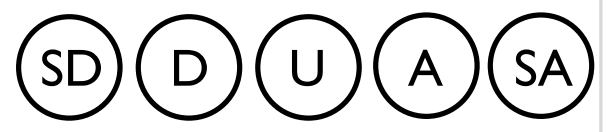

3 I think feedback about my progress during cardiac rehabilitation would be helpful

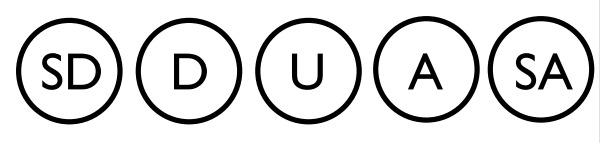

4 I do not think community-based cardiac rehabilitation is very safe

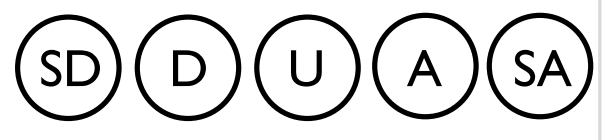

5 I sometimes feel depressed about the state of my

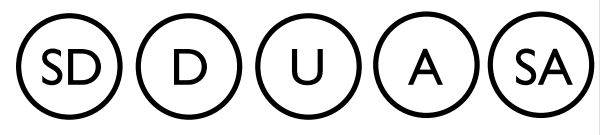

6 I would like to learn more about how to improve my health

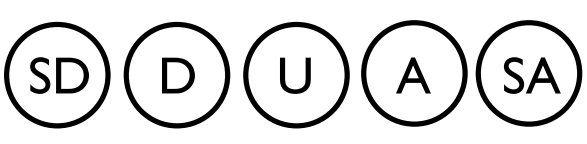

7 I think cardiac rehabilitation might do more harm than good

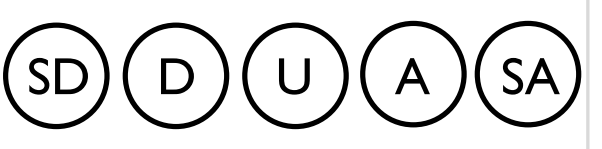

8 I sometimes feel awkward when meeting new people

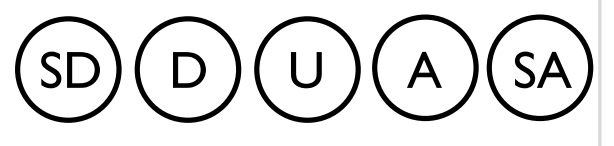

9 I like the idea of meeting other people at cardiac

rehabilitation who have similar health problems to me

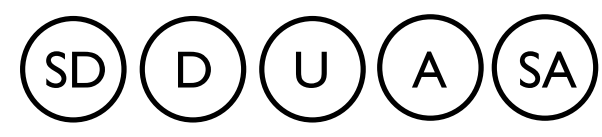

10 Cardiac rehabilitation will probably be quite disruptive to my life

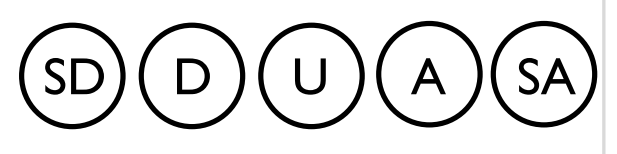

I I I feel a bit self-conscious about my physical appearance

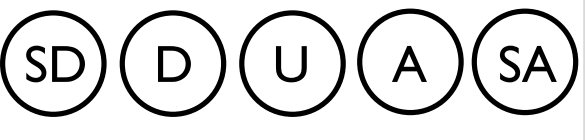

I2 I would like to have a more active lifestyle

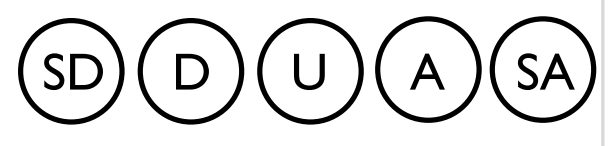




I5 I am usually comfortable in a group environment
I4 Coctor recommended it
I6 I don't really understand how cardiac rehabilitation
works

Administrative use:

\begin{tabular}{|c|c|c|c|c|c|c|}
\hline Item & Score & Item & Score & Item & & Score \\
\hline$I$ & $=$ & 2 & $=$ & 3 & $=$ & \\
\hline 4 & $=$ & 5 & $=$ & 6 & $=$ & \\
\hline 7 & $=$ & 8 & $=$ & 9 & $=$ & \\
\hline 10 & $=$ & 11 & $=$ & 12 & $=$ & \\
\hline 13 & $=$ & 14 & $=$ & 15 & $=$ & \\
\hline 16 & $=$ & 17 & $=$ & 18 & $=$ & \\
\hline OA & $=$ & PA & $=$ & $\mathbf{A}$ & $=$ & \\
\hline
\end{tabular}


Once a respondent has completed the CRI, the score for each individual question can be recorded in the column labelled 'admin use' located immediately to the right of the Likert response matrix. All items should be scored using the following system:

Strongly Disagree $=0 ;$ Disagree $=1 ;$ Undecided $=2 ;$ Agree $=3 ;$ Strongly Agree $=4$

Scores should be inserted into the table at the end of the CRI and then each column added up to produce an overal score for outcome anxiety (OA), process anxiety (PA) and autonomy (A). Process anxiety is equal to the sum of items $1,4,7,10,13,16$ and 19. Outocme anxiety is equal to the sum of items $2,5,8,1 \mathrm{I}, 14,17$ and 20 . Autonomy is equal to the sum of items $3,6,9,12,15$, 18 and 21 .

Interpreting CRI Scores:

The range of scores available for each component (PA, OA \& A) is 0 to 24. Lower PA and OA scores are desirable whereas higher $A$ scores are desirable. The CRI responses of $4 I 2$ cardiac rehabilitation patients indicates an interquartile range of $16-2 \mid$ for process anxiety, I3-18 for outcome anxiety and 16-19 for autonomy. Individuals who score in the upper quartile for process anxiety (2I or greater), in the upper quartile for outcome anxiety (I 8 or greater) and in the lower quartile for autonomy ( 16 or less) may be at particular risk of disengaging with cardiac rehabilitation and may benefit from special help in tailoring their programme.

Those with high outcome anxiety may be reluctant to participate in CR because of feelings of vulnerability or irrational fears that $C R$ will exacerbate their prognosis. It may be advantageous for such individual's to begin CR by attending education sessions before any involvement in exercise in an attempt to redress irrational beliefs and assuage their anxiety.

Those with high process anxiety may feel unfamiliar, uncomfortable or intimidated by the group or exercise environment associated with CR. Such individuals may benefit from having a progressive introduction to $C R$ that perhaps first involves watching an exercise session, meeting other CR participants in a non-exercising context and having a mentor who is an existing participant. It may also be beneficial to take into consideration their individual preferences with respect to types of exercise and gender groups.

Those who have low autonomy scores are likely to have a high external locus of control resulting in greater dependency on others and passive engagement in CR. In some instances it will be possible to address low autonomy by simply helping with the practical matters such as CR travel, timing, location and subsidisation. For other individual's, a lack of autonomy might be improved by involving them in decision-making processes about their CR, offering them choice and then getting them to reflect on the outcomes of their choices.

These suggestions of how to address high anxiety and low autonomy are by no means exhaustive but rather examples of how practitioners might use CRI information to provide appropriate levels of individual support. The overarching principle is to tailor support accord to individual need and the CRI can provide some of the information needed in this process. 